\title{
Catalyzing Collaborative Communities: the ABCs
}

\author{
ARIEL ANBAR \\ Arizona State University \\ Presenting Author: anbar@asu.edu
}

Science is increasingly collaborative, but early career training is organized around independent apprenticeship, and promotion in academia is based on individual metrics. Consequently, academic scientists rarely experience effective collaborative cultures or receive guidance in their creation. Important principles emerge when examining successful collaborations and institutions, including:

A: Advance Alliances. An "alliance" signifies shared goals of mutual benefit to all individuals. Progress toward goals becomes a metric against which success is measured, transforming a group that shares interests into a functional community of individuals who feel compelled to connect. While seemingly obvious, effectively advancing alliances requires sustained planning and organization, accountability of leaders to the group, inclusive norms, and cultivation of collective buy-in to goals and milestones. Collaborative research projects are the most obvious example, but diverse goals are possible. Increased awareness of social justice challenges within academia presents novel avenues for compelling, goal-directed efforts.

B: Build Bonds. Academics dedicate their lives to advancing knowledge through reason and so it is natural for them to imagine that humans are fundamentally rational. A large and growing body of research, and long experience, shows otherwise. This realization has important implications for how communities should be organized and managed. In particular, social opportunities and group experiences, often viewed by academic culture as fun but disposable, distracting "extras", need to be recognized as essential and even central, demanding primacy of time, space, funding, and planning effort. "Fun" needs to be taken seriously!

C: Center the Students (and postdocs). Overcoming individual self-interest is a fundamental challenge to forming functional collaborative communities. A highly effective strategy in academia is to center activities around research and training opportunities for early career scientists. When students and postdocs pursue projects that require mentoring by multiple members of the community, collaborations can form organically in which established scientists subordinate their immediate interests to communal goals. However, fostering and sustaining such a student-centered culture is not organic. It requires careful attention by leaders to funding distribution, accountability mechanisms, and behavioral norms.

This keynote will review examples and foster discussion around these and other principles. 